\section{Measuring the learning-relevant relations between variables in experience}

\author{
K. RICHARDSON \\ Faculty of Educational Studies, The Open University \\ Walton, Bletchley, Milton Keynes MK7 6AA, England
}

In recent years, attempts have been made to understand natural phenomena in terms of the relations between constituent variables instead of as clusters of independent attributes. In human concept learning, for instance, considerable attention has been devoted to the nature of the relations between instances defining a category or concept (e.g., Medin \& Schwanenflugel, 1981; Mervis \& Rosch, 1981), although this has not, as yet, issued in any formal expression or quantitative measure. Similar considerations have arisen about the ecology and evolution of behavior. Thus, Plotkin and Odling-Smee (1979) describe learning as an evolutionary response to environmental unpredictability, or, more exactly, as a device that can extract predictability from uncertainty and change. Colwell (1974), on the other hand, has clearly shown that such predictability can be understood only in terms of the relations between variables defining a phenomenon and uses a metric derived from the Shannon information statistic to measure the predictability in seasonal or cyclical phenomena. Richardson and Bhavnani (1982) use a sometime different metric, derived from Kullback (1968), to define and measure the relation between variables entering into human concept formation. This measure may have more general applicability.

The measure will be illustrated here, first of all, with the relations among a simple set of variables. Suppose a natural phenomena manifests itself in the form of three variables, each of three levels, for example, a sample from a population of objects of three sizes, three shapes, and three colors. The probability of occurrence of some experienced combinations (objects) is, however, greater than that of others. What we want to know is the joint relation between these variables such that we can tell the extent to which any particular size predicts shape and color, and so on. A simple way of representing this experience is in the form of a three-way contingency table in which the probabilities are entered in cells. If we let pijk be the probability of occurrence in the ith row, jth column, and $k$ th depth, and:

$$
P i . .=\sum_{j=1}^{c} \sum_{k=1}^{d} P i j k
$$

$$
\text { P.j. }=\sum_{i=1}^{r} \sum_{k=1}^{d} P i j k
$$

and

$$
P . k=\sum_{i=1}^{r} \sum_{j=1}^{c} P i j k .
$$

Then, according to the analysis of Kullback (1968, see p. 156):

$$
I=\sum_{i} \sum_{j} \sum_{k} P i j k \log \left(P_{i j k} / \text { Pi..P.j.P..k. }\right),
$$

where $I$ is a measure of the relation between the row, column, and depth categories and has also been defined as the mean information in any one category about the other categories (Kullback, 1968, p. 156).

This measure is easily extended to higher order relations, the only obstacle being algebraic complexity, and this is easily overcome with the use of any microcomputer capable of matrix functions. The only restriction, then, is the capacity of the machine. For instance, a five-way matrix is easily accommodated in a machine of $32 \mathrm{~KB}$, but higher order relations probably require more memory, depending on the number of levels of each variable.

The program first uses input variables and respective levels to dimension an array. Probabilities corresponding to particular cells may then be entered from the keyboard or assigned in the program. Each pi.. is calculated using FOR-NEXT commands for the js and ks (assuming a three-way matrix), and similarly for the p.j.s and p..ks. It is then a fairly straightforward matter to calculate I using Equation 1. A further advantage of the measure is that, once $I$ has been obtained, manipulation of Equation 1 permits the direct, quantitative prediction, in terms of probability of occurrence, of any novel combinations. This should prove useful in further studies in concept learning, in which transfer of learning to new instances is a key test (Smith \& Medin, 1981).

A program listing in PET BASIC for three- or fiveway matrix calculations is available from the author. A copy, for use with the PET, can be obtained upon submission of a blank tape.

\section{REFERENCES}

Colwell, R. L. Predictability, constancy and contingency of periodic phenomena. Ecology, 1974, 55, 1148-1153.

Kullback, S. Information theory and statistics. New York: Dover, 1968. 
Medin, L. D., \& Schwanenfluged, P. J. Linear separability in classification learning. Journal of Experimental Psychology: Human Learning and Memory, 1981, 7, 355-368.

Mervis, C. B., \& Rosch, E. E. Categorization of natural objects. Annual Review of Psychology, 1981, 32, 89-115.

Plotkin, H. C., \& Odling-Smee, F. J. Learning, change and evolution: An enquiry into the teleonomy of learning. Advances in the Study of Behaviour, 1979, 10, 1-42.
Rtchandson, K., \& Bhavnan1, K. K. Abstraction of contingency in concept learning. Current Psychological Research, 1982, 4, $1-9$.

Smith, E. E., \& Medin, D. L. Categories and concepts. Cambridge, Mass: Harvard University Press, 1981.

(Accepted for publication November 10, 1982.) 